\title{
Hypoxic culture and expansion of mesenchymal stem cells in airlift loop hollow fiber membrane bioreactor
}

\author{
Xiangqin $\mathrm{Li}^{1}$, Tianqing Liu ${ }^{1}$, Kedong Song ${ }^{1}$, Xuehu Ma ${ }^{1}$, Zhanfeng $\mathrm{Cui}^{2}$ \\ ${ }^{I}$ Stem Cell and Tissue Engineering Lab of Dalian University of Technology, Dalian, China; ${ }^{2}$ Oxford Centre for Tissue Engineering \\ and Bioprocessing, Department of Engineering Science, Oxford University, Oxford OX1 3PJ, UK
}

Mesenchymal stem cells (MSCs), having the multi-differentiation potential of becoming a variety of cells, including fat, cartilage, bone, tendon and ligaments, muscle, skin cells and even nerve cells, are the promising "seeding cells" for tissue engineering. Besides, MSCs are excellent tools for gene therapy. However, MSCs are in limited quantity and their viability decreases with the individual age increases, which makes it necessary for them to be expanded in vitro. Many researches indicated that hypoxia accelerated MSCs proliferation and differentiation, but all were in two dimension and static conditions. So in this paper, MSCs were cultured and expanded in hypoxic, threedimensional and dynamic conditions in vitro. MSCs of passage $4(\mathrm{P} 4)$ mixed with $3 \mathrm{mg} \cdot \mathrm{mL}^{-1}$ type I collagen with bFGF of $5 \mathrm{ng} \cdot \mathrm{mL}^{-1}$ at the density of $1 \times 10^{5}$ cells $\cdot \mathrm{mL}^{-1}$ were innoculated into hollow fiber membranes (HFM), where the gel with MSCs formed after an hour in an incubator at $37{ }^{\circ} \mathrm{C}, 5 \% \mathrm{CO}_{2}, 5 \% \mathrm{O}_{2}$ and saturated humidity and then was innoculated into an air-lift loop hollow fiber membrane bioreactor (ALHFMB) for the dynamic culture in the incubator. During the experiments, the OD value of medium was detected every $24 \mathrm{~h}$ to calculate cell number and to obtain cell growth curves, and the metabolic parameters, such as glucose, lactic acid, ammonia, glutamine, $\mathrm{pH}$, $\mathrm{O}_{2}$ and $\mathrm{CO}_{2}$ were also detected to evaluate the cells growth. Moreover, the expanded cells were identified through antibodies CD29, CD44 and CD45 by flow cytometer and through inducing them into osteoblasts, chondrocytes and adipocytes. The results showed that in the ALHFMB, $\mathrm{O}_{2}$ concentration kept constant; MSCs metabolized robustly and expanded about 50-fold within 7 days in hypoxic condition, more than that in normoxic conditions; most of the expanded cells were CD29 and CD44 positive and CD45 negative; after being induced, the expanded cells still reserved the strong muti-differentiation potential of becoming bone, cartilage and adipose. This study first explored the feasibility of 3D, dynamic and hypoxic expanding MSCs in ALHFMB, and the expanded cells still keep the capacity of extensive self-renewal and multilineage differentiations. Therefore, it is a safe protocol for MSCs to be cultured inside HFM with type I collagen for hypoxic expansion.

Keywords: mesenchymal stem cells, hypoxia, hollow fiber membrane, bioreactor, gel

Cell Research (2008) 18:s169. doi: 10.1038/cr.2008.259; published online 4 August 2008

Correspondence: Xiangqin Li

E-mail: xiangqinli@163.com 